\title{
Modelo de predicción de aislamiento acústico para paneles simples y multicapa
}

\section{Sound insulation prediction model for simple and multilayer panels}

\author{
Joaquín Mansilla $^{\# 1}$, Laurence Bender ${ }^{* 2}$, Leonardo Pepino ${ }^{\# 3}$ \\ \# Ingeniería de Sonido, Universidad Nacional Tres de Febrero, Mosconi 2736, Saenz Peña, Argentina. \\ 1 jmansilla@untref.edu.ar \\ 3 leonardodpepino@gmail.com \\ * Laboratorio de Arte Electrónico e Inteligencia Artificial, Universidad Nacional Tres de Febrero, Valentín Gómez 4692, \\ Caseros, Argentina. \\ 2 lbendereuntref.edu.ar
}

Recibido: 29/10/18; Aceptado: 25/04/19

\begin{abstract}
Airborne acoustic insulation prediction models allow to evaluate the sound transmission loss of construction solutions in buildings. Among the different building materials used in constructions, homogeneous and isotropic materials of various thicknesses, such as concrete and glass, are commonplace. Due to the limitations of simple panels made from materials of standard thickness, in order to increase the acoustic insulation it is necessary to add various layers of the same or different materials. This research presents a model based on several approximations depending on the frequency range and comprises multi-layer panels joined together using "spot" techniques, such as by screws or nails. The model performance is evaluated with a comparison between predictions obtained using the commercial software INSUL and the model proposed by the authors. The predicted values are also compared with laboratory measurements obtained according to ISO 140-3. This research considers panels grouped into two categories of materials: light and heavyweight materials. The transmission loss in third octave bands, the weighted sound reduction indices and the spectral adaptation terms Rw (C,Ctr) are examined. Statistically, the results show that the proposed model agrees well with real measurements, both for single and multi-layer panels.
\end{abstract}

Keywords: sound insulation; prediction model; laminated panels.

Resumen - Los modelos de predicción de aislamiento acústico son una herramienta importante a la hora de diseñar los elementos constructivos que integran una obra de construcción. Existen materiales homogéneos e isotrópicos de diferentes espesores como el hormigón o el vidrio, entre otros. En muchos casos para alcanzar el aislamiento acústico requerido se suele superponer varias capas del mismo o diferentes materiales para aumentar la masa superficial del sistema. En esta investigación se presenta un modelo basado en diferentes aproximaciones según el rango de frecuencias para calcular el índice de reducción sonora $R$ en el caso de paneles simples y paneles multicapa unidos de forma puntual, como puede ser mediante tornillos o clavos. Se evalúa el desempeño del modelo mediante una comparativa entre las predicciones obtenidas utilizando el programa comercial INSUL y las obtenidas con el modelo propuesto. A su vez, se contrastan los valores con mediciones en laboratorio realizadas de acuerdo a la norma ISO 140-3. El estudio considera paneles agrupados en dos categorías de materiales: livianos y pesados. Se examinan las pérdidas por transmisión en bandas de tercio de octava, los índices de reducción sonora ponderados y los términos de adaptación espectral $\operatorname{Rw}(\mathbf{C}, \mathrm{Ctr})$. Estadísticamente, los resultados muestran que el modelo propuesto se adapta a los ensayos disponibles, tanto para paneles simples como para multicapa.

Palabras clave: aislamiento acústico; modelo predicción; panel multicapa.

\section{INTRODUCCIÓN}

La transmisión de ondas a través de una partición divisoria entre dos recintos depende de las características físicas de la solución constructiva. Para paneles simples, homogéneos e isotrópicos, las variables relevantes a tener en cuenta para la predicción del grado de aislamiento acústico son el módulo de Young $E$ del material del panel, el coeficiente de Poisson $v$, el factor de pérdidas interno $\eta$, la masa superficial $m$ que se calcula a partir de la densidad del material $\rho$ multiplicada por el espesor del panel $h$, la frecuencia $f$ y el ángulo de incidencia de la onda.

La predicción del índice de reducción sonora $R$, que expresa la relación energética en forma logarítmica entre la onda transmitida respecto a la onda incidente, ha sido un tema de estudio tratado desde hace tiempo. Jäeger [1], Lord Rayleigh [2], Berger [3] y Wintergerst [4] fueron los primeros en hablar del fenómeno de aislamiento acústico entre finales del siglo XIX y principios del siglo $\mathrm{XX}$. Cremer [5] en el año 1942 presentó un modelo de predicción para paneles simples e isotrópicos y mostró la importancia del efecto de coincidencia de las ondas de flexión.

Desde aquel estudio hasta la actualidad existe una extensa bibliografía sobre modelos de predicción de paneles simples de una sola hoja. Investigaciones como la de London [6], Josse y Lamure [7], Crocker y Pryce [8], Sewell [9], Brekke [10], hasta Sharp [11], [12], Arau [13] o Davy [14], [15], han tratado de modelar el comportamiento vibroacústico desde distintos puntos de vista, sea mediante modelos de impedancia, análisis energético estadístico SEA, o mediante analogías electro-mecano-acústicas, entre otros. La mayoría de los investigadores se basan en modelos diferentes según si la frecuencia a aislar es menor o mayor a 
la crítica $f_{c}$. Se define a la frecuencia crítica como aquella donde se iguala la velocidad de propagación de las ondas de flexión $c_{F}$ en el panel a la velocidad de propagación del sonido en el aire $c_{0}$.

En la práctica, una solución ampliamente utilizada en la construcción es la superposición de distintas capas del mismo o distinto material para aumentar las pérdidas de transmisión. Estos casos se pueden tratar a partir de las ecuaciones de movimiento de una placa delgada y del modelo de predicción de paneles múltiples por método de impedancias, [11], [16], [17], asumiendo que el espesor de la cavidad entre paneles tiende a cero.

Estos desarrollos han permitido crear herramientas de cálculo para predecir el comportamiento de soluciones constructivas y poder así, por ejemplo, cumplir con las exigencias de aislamiento a ruido aéreo que requiera la reglamentación de cada país para la protección de los ocupantes en los edificios. Entre los programas comerciales de predicción más conocidos se encuentran INSUL [18], dBKaisla [19], ENC [20] y SoundFlow [21], entre otros.

INSUL es uno de los programas de predicción más utilizados en el ámbito de la acústica arquitectónica [22]. Los modelos de predicción que utiliza para paneles de una hoja se basan en los modelos analíticos de Cremer y Sharp, entre otros, y en modelos empíricos, según los creadores del programa [18]. Sin embargo, el código fuente de INSUL es cerrado y no se brindan detalles sobre la implementación de los modelos y las ecuaciones utilizadas. En el caso de paneles multicapa no se brinda ninguna información sobre el modelo de predicción y su funcionamiento.

El objetivo de este trabajo de investigación es evaluar el modelo de predicción propuesto por los autores mediante una comparativa con el programa INSUL versión 9 y ensayos en condiciones de laboratorio disponibles en la bibliografía [23]-[26].

Se analizan las desviaciones de los índices de reducción sonora $R$ por bandas de frecuencia en tercio de octava respecto a mediciones y los índices globales $R w(C, C t r)$ calculados según la norma ISO 717-1 [27]. Se utilizaron bases de datos con mediciones de laboratorio que cumplen la norma ISO 140-3 [28].

Primero se presentan las ecuaciones del modelo propuesto, considerando si la predicción se realiza para frecuencias menores o mayores con respecto a la frecuencia crítica equivalente $f_{c, e q}$, sea de un panel simple o multicapa. Se comparan por separado paneles simples de una sola capa y paneles multicapa de placas de yeso. En el caso de paneles simples se divide la comparativa en dos grupos de materiales: livianos y pesados. Se discuten los resultados obtenidos de la comparativa con el fin de validar el modelo propuesto. Finalmente se presentan las conclusiones de la investigación.

\section{Modelo de PRedicción Propuesto}

A partir del análisis previo de distintos métodos de predicción [29] se propone un modelo de aislamiento acústico, tanto para paneles simples homogéneos e isotrópicos como sistemas multicapa compuestos por paneles simples de distintos materiales y espesores. En este caso se considera que las capas se encuentran unidas entre sí mediante fijaciones puntuales y no adheridas en toda su superficie. El modelo propuesto utiliza una extensión de las ecuaciones presentadas por Davy [14], [15] finitas para frecuencias menores a la frecuencia crítica equivalente de la estructura multicapa $\left(f<f_{c, e q}\right)$. En este rango de frecuencias el modelo considera que el panel es de dimensiones finitas. En las frecuencias mayores e iguales a la frecuencia crítica equivalente $\left(f \unlhd f_{c, e q}\right)$ se utiliza una extensión del modelo propuesto por Sharp [11], [12].

\section{A. Modelo para frecuencias menores a la frecuencia crítica equivalente}

Para la predicción de $R$ en el caso de frecuencias menores a la frecuencia crítica el modelo propuesto se basa en el trabajo desarrollado por Davy, quien define el coeficiente de transmisión en campo difuso $\tau_{d}$ como

$$
\tau_{d}=2 \int_{0}^{\frac{\pi}{2}} \tau(\theta) \cos \theta \sin \theta d \theta,
$$

donde $\tau(\theta)$ es el coeficiente de transmisión en función del ángulo de incidencia $\theta$ y el factor 2 es un factor de normalización.

El modelo de Davy tiene en cuenta el tamaño finito del panel a partir de la eficiencia de radiación por ondas de flexión $\sigma(\theta)$ [30]. En el caso de un panel infinito este factor se aproxima con la siguiente expresión

$$
\sigma(\theta)=\frac{1}{\cos \theta} .
$$

Para el caso de un panel de dimensiones finitas, Davy define la eficiencia de radiación como

$$
\sigma(\theta)=\left\{\begin{array}{lll}
\frac{1}{\frac{2 \pi c_{0}^{2}}{\omega^{2} A}+\cos \theta} & \text { si } & |\theta| \leq \varphi \\
\frac{1}{\frac{2 \pi c_{0}^{2}}{\omega^{2} A}+\frac{1}{2}(3 \cos \varphi-\cos \theta)} & \text { si } & \varphi<|\theta| \leq \frac{\pi}{2}
\end{array}\right.
$$

donde $\varphi$ toma los siguientes valores,

$$
\varphi= \begin{cases}0 & \text { si } \sqrt{\frac{\pi c_{0}}{\omega \sqrt{A}}} \geq 1 \\ \cos ^{-1}\left(\sqrt{\frac{\pi c_{0}}{\omega \sqrt{A}}}\right) & \text { si } \sqrt{\frac{\pi c_{0}}{\omega \sqrt{A}}}<1,\end{cases}
$$

siendo $\omega$ la frecuencia angular en $\mathrm{rad} / \mathrm{s}, c_{0}$ la velocidad de propagación del sonido en el aire en $\mathrm{m} / \mathrm{s}$ y $A$ la superficie del panel en metros cuadrados.

Partiendo de las ecuaciones (1) y (2) se puede reescribir la ecuación del coeficiente de transmisión difuso $\tau_{d}$ considerando la eficiencia de radiación como

$$
\tau_{d}=2 \int_{0}^{\frac{\pi}{2}} \frac{\tau(\theta)}{\sigma(\theta)} \sin \theta d \theta .
$$

Substituyendo el factor de radiación $\sigma(\theta)$ de la ecuación (3) en la ecuación (5) y el coeficiente de transmisión en función del ángulo de incidencia [5], 


$$
\tau(\theta)=\left|\frac{p_{t}}{p_{i}}\right|^{2}=\left|\frac{1}{1+\frac{Z_{T}}{2 \rho_{0} c_{0} \sigma(\theta)}}\right|^{2},
$$

donde $p_{t}$ y $p_{i}$ son la presión acústica transmitida e incidente respectivamente en $\mathrm{Pa}, \theta$ es el ángulo de incidencia y $\rho_{0}$ es la densidad del aire en $\mathrm{kg} / \mathrm{m}^{3}$.

El término $Z_{T}$ es la impedancia mecánica total por unidad de superficie. El modelo que se propone en esta investigación considera que para un sistema multicapa conformado por paneles simples unidos entre sí de forma puntual, $Z_{T}$ se puede expresar como

$$
Z_{T}=\sum_{i=1}^{N} Z_{i},
$$

donde $Z_{i}$ es la impedancia mecánica por unidad de superficie de la i-ésima lámina de la estructura multicapa y $N$ el número de capas. A partir de la teoría de vibración de placas, la impedancia $Z_{i}$ se puede escribir como

$$
Z_{i}=j\left(m_{i} \omega-B_{i} \frac{\omega^{3}}{c_{0}^{4}} \sin ^{4}(\theta)\right)+B_{i} \eta_{i} \frac{\omega^{3}}{c_{0}^{4}} \sin ^{4}(\theta) .
$$

Substituyendo (8) en (7), $Z_{T}$ se puede reescribir de la siguiente forma,

$$
\begin{aligned}
& Z_{T}=j\left(\omega \sum_{i=1}^{N} m_{i}-\sum_{i=1}^{N} B_{i} \frac{\omega^{3}}{c_{0}^{4}} \sin ^{4}(\theta)\right)+\sum_{i=1}^{N}\left(B_{i} \eta_{i}\right) \frac{\omega^{3}}{c_{0}^{4}} \sin ^{4}(\theta)= \\
& =\omega \sum_{i=1}^{N} m_{i}\left[j\left(1-\frac{\sum_{i=1}^{N} B_{i}}{\sum_{i=1}^{N} m_{i}} \frac{\omega^{2}}{c_{0}^{4}} \sin ^{4}(\theta)\right)+\frac{\sum_{i=1}^{N}\left(B_{i} \eta_{i}\right)}{\sum_{i=1}^{N} m_{i}} \frac{\omega^{2}}{c_{0}^{4}} \sin ^{4}(\theta)\right],
\end{aligned}
$$

donde $m_{i}$ es la masa superficial en $\mathrm{kg} / \mathrm{m}^{2}, B_{i}$ es la rigidez a la flexión y $\eta_{i}$ es el factor de pérdidas total de la capa iésima. Este factor está compuesto por el factor de pérdidas interno del propio material $\eta_{i n t, i}$ más el factor de pérdidas por efecto borde $\eta_{e d g e, i}$ [14], el cuál según la norma ISO 12354-1 [31] se puede expresar de la siguiente forma,

$$
\eta_{i}=\eta_{\text {int }, i}+\eta_{\text {edge }, i} \cong \eta_{\text {int }, i}+\frac{m_{i}}{485 \sqrt{f}} .
$$

La rigidez a la flexión $B_{i}$ se obtiene a partir del módulo de Young $E_{i}$, el coeficiente de Poisson $v_{i}$ y el espesor $h_{i}$ del panel de la siguiente forma,

$$
B_{i}=\frac{E_{i} h_{i}^{3}}{12\left(1-v_{i}^{2}\right)} .
$$

Por otro lado, la frecuencia crítica de cada una de las capas se obtiene a partir de su rigidez a la flexión, su masa superficial y la velocidad de propagación de la onda acústica en el aire, de forma que,

$$
f_{c_{i}}=\frac{c_{0}^{2}}{2 \pi} \sqrt{\frac{m_{i}}{B_{i}}}
$$

De manera análoga, la frecuencia crítica equivalente de la estructura multicapa se puede expresar como

$$
f_{c, e q}=\frac{c_{0}^{2}}{2 \pi} \sqrt{\frac{\sum_{i=1}^{N} m_{i}}{\sum_{i=1}^{N} B_{i}}} .
$$

Substituyendo (13) en la expresión (9),

$$
Z_{T}=\omega \sum_{i=1}^{N} m_{i}\left[\begin{array}{c}
j\left(1-\frac{f^{2}}{f_{c, e q}^{2}} \sin ^{4}(\theta)\right)+ \\
\frac{\sum_{i=1}^{N}\left(B_{i} \eta_{i}\right)}{\sum_{i=1}^{N} B_{i}} \frac{f^{2}}{f_{c, e q}^{2}} \sin ^{4}(\theta)
\end{array}\right]
$$

A su vez, se puede definir la relación entre frecuencia y frecuencia crítica equivalente de la siguiente forma,

$$
r=\frac{f}{f_{c, e q}} .
$$

Substituyendo entonces (15) en la expresión (14), la impedancia total $Z_{T}$ se puede expresar como

$$
Z_{T}=\omega \sum_{i=1}^{N} m_{i}\left[j\left(1-r^{2} \sin ^{4}(\theta)\right)+\frac{\sum_{i=1}^{N}\left(B_{i} \eta_{i}\right)}{\sum_{i=1}^{N} B_{i}} r^{2} \sin ^{4}(\theta)\right] .
$$

Finalmente, para calcular el índice de reducción sonora $R$ expresado en decibeles para $f<f_{c, e q,}$,

$$
R=-10 \log \left(\tau_{d}\right),
$$

donde la integral (5) se evalúa numéricamente.

\section{B. Modelo para frecuencias mayores o igual a la frecuencia crítica}

La expresión de $R$ del modelo de Sharp [8] para $f \unlhd f_{c}$ de un panel de una sola capa se calcula a partir de comparar frecuencia a frecuencia $R_{1}$ y $R_{2}$ y elegir el resultado menor:

$$
\begin{gathered}
R_{1}=10 \log \left(1+\left(\frac{\pi m f}{\rho_{0} c_{0}}\right)^{2}\right)+10 \log \left(\frac{2 \eta f}{\pi f_{c}}\right) \\
R_{2}=10 \log \left(1+\left(\frac{\pi m f}{\rho_{0} c_{0}}\right)^{2}\right)-5,5 .
\end{gathered}
$$

Al considerar un panel formado por múltiples capas superpuestas, no adheridas, las expresiones (18) y (19) se modifican. Siguiendo un análisis similar al desarrollado en [15] usando la expresión de la impedancia mecánica total por unidad de superficie (16) para $r>2$, las ecuaciones se pueden reescribir de la siguiente manera para $f \geq f_{c, e q}$,

$$
\begin{gathered}
R_{1}=10 \log \left(1+\left(\frac{\pi \sum_{i=1}^{N}\left(m_{i}\right) f}{\rho_{0} c_{0}}\right)^{2}\right)+10 \log \left(\frac{2 \sum_{i=1}^{N}\left(B_{i} \eta_{i}\right) f}{\sum_{i=1}^{N}\left(B_{i}\right) \pi f_{c, e q}}\right), \\
R_{2}=10 \log \left(1+\left(\frac{\pi \sum_{i=1}^{N}\left(m_{i}\right) f}{\rho_{0} c_{0}}\right)^{2}\right)-5,5 .
\end{gathered}
$$

\section{RESULTADOS}

\section{A. Comparativa de paneles simples de una capa}

Para evaluar el desempeño del modelo propuesto, primero se realiza una comparativa para paneles simples respecto a cuatro materiales con distintos espesores. 

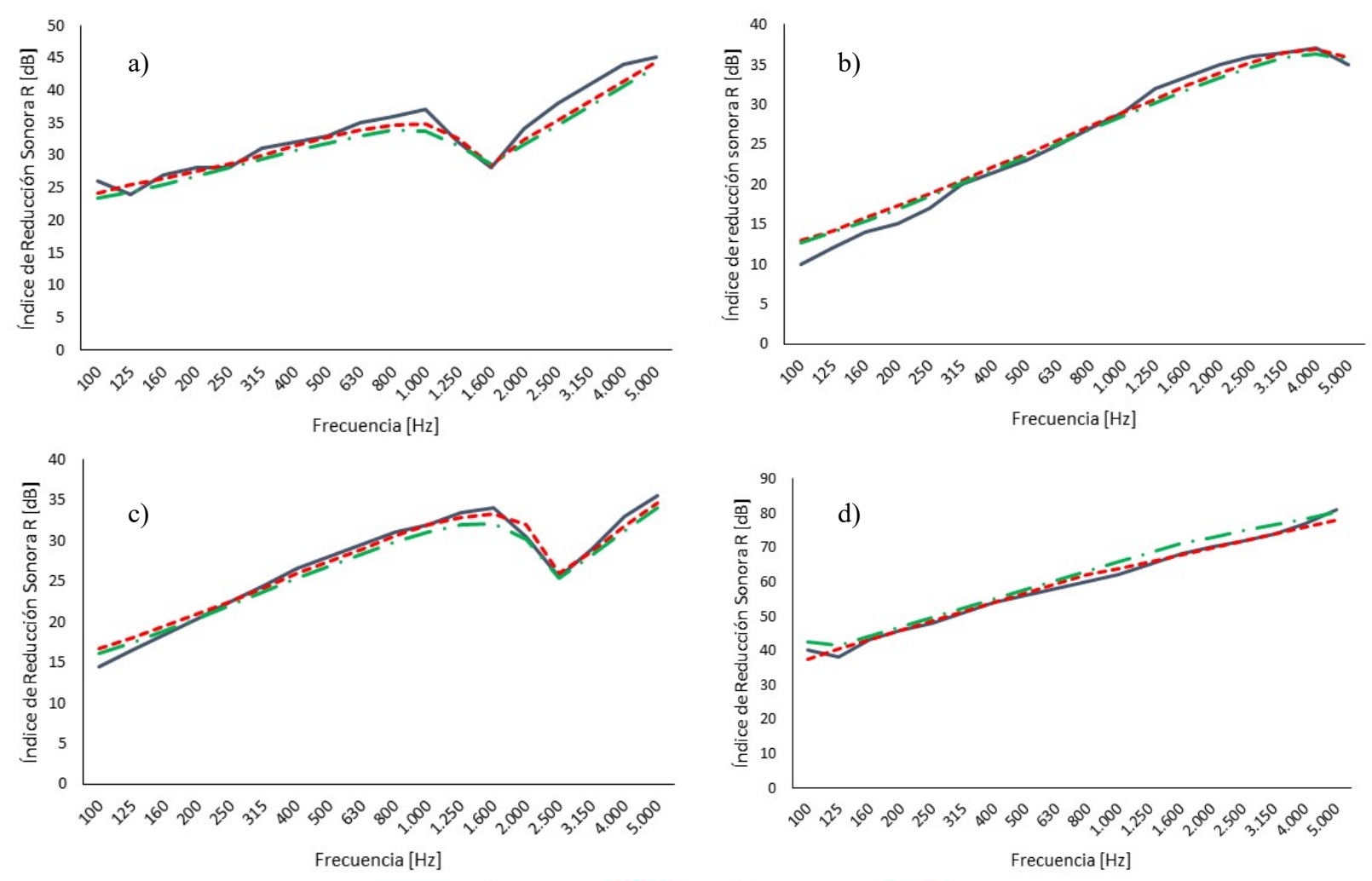

Laboratorio _- Modelo propuesto - - INSUL

Fig 1. Comparativa de los índices de reducción sonora entre la medición de laboratorio, la predicción del modelo propuesto y el programa INSUL para: a) vidrio de $8 \mathrm{~mm}$, b) madera $\mathrm{HDF}$ de $6,4 \mathrm{~mm}$, c) placa de yeso de $15,9 \mathrm{~mm}$ y d) hormigón de $180 \mathrm{~mm}$.

Solamente se han considerado referencias con mediciones en bandas de tercio de octava obtenidas mediante laboratorio según norma ISO 140-3 [23]-[26]. Los materiales utilizados con sus respectivas características físicas se muestran en la Tabla 1. En el caso del hormigón y el vidrio los ensayos no ofrecen estos datos, por lo que se optó por utilizar los valores disponibles en la base de datos del programa INSUL. En el caso del vidrio se ha considerado una altura de la muestra de 0,9 metros por 2,1 metros de ancho, y para el resto de materiales una altura de 3 metros por 4 metros de ancho. Se han analizado un total de 22 mediciones. También se realiza la misma comparativa con respecto a los resultados arrojados por el modelo de predicción del programa INSUL en su versión 9, a fin de contrastar los resultados con los del modelo propuesto.

En la Fig. 1 se muestran los índices de reducción sonora $R$ en bandas de tercio de octava de distintos materiales de un espesor determinado, comparando los resultados obtenidos mediante ensayos y las predicciones obtenidas con el programa INSUL y con el modelo propuesto.

\section{B. Comparativa paneles multicapa}

Para evaluar la adaptación del modelo propuesto para el caso de paneles multicapa, se han estudiado cuatro mediciones disponibles de distintas combinaciones de placas de yeso [21]. Los espesores en milímetros son las siguientes: $6,4+6,4,12,7+12,7,12,7+15,9,15,9+12,7+15,9$. Para las primeras dos combinaciones la distancia entre montantes, donde se fijan los paneles, es de 12 pulgadas, y para el tercer y cuarto caso la separación es de 24 pulgadas.
TABLA I

LISTA DE MATERIALES UTILIZADOS EN LA COMPARATIVA Y SUS CARACTERÍSTICAS FÍSICAS

\begin{tabular}{|c|c|c|c|c|c|}
\hline Material & $\begin{array}{c}\text { Espesor } \\
{[\mathbf{m m}]}\end{array}$ & $\begin{array}{c}\text { Densidad } \\
{\left[\mathbf{K g} / \mathbf{m}^{3}\right]}\end{array}$ & $\begin{array}{c}\text { Módulo } \\
\mathbf{d e} \\
\text { Young } \\
{\left[\mathbf{G N} / \mathbf{m}^{2}\right]}\end{array}$ & $\begin{array}{c}\text { Factor } \\
\text { Coeficiente } \\
\text { de Poisson }\end{array}$ & $\begin{array}{c}\text { de } \\
\text { pérdidas } \\
\text { interno }\end{array}$ \\
\hline Hormigón & $\begin{array}{c}50,8 ; \\
101,6 ; \\
140 ; \\
160(\mathrm{x} 2) ; \\
180 ; \\
200 ; \\
220 ; \\
240\end{array}$ & 2100 & 30 & 0,2 & 0,03 \\
\hline Vidrio & $\begin{array}{c}3 ; 4 ; 6 ; \\
8 ; 10 ; \\
12 ; 19\end{array}$ & 2500 & 71 & 0,23 & 0,02 \\
\hline Placas de & $\begin{array}{c}6,4 ; 9,5 ; \\
12,7 ; \\
\text { Yeso }\end{array}$ & 76,9 & 2 & 0,23 & 0,01 \\
\hline HDF & 3,$2 ; 6,4$ & 1250 & 3 & 0,15 & 0,03 \\
\hline
\end{tabular}

Las características físicas de este material se presentan en la Tabla 1. Se ha considerado paneles de 3 metros de altura por 4 metros de ancho. En la Fig. 2 se muestran las curvas del índice $\mathrm{R}$ en bandas de tercio de octava, donde en cada subfigura se muestra la curva de la medición en laboratorio, los resultados del modelo propuesto y del programa INSUL. 

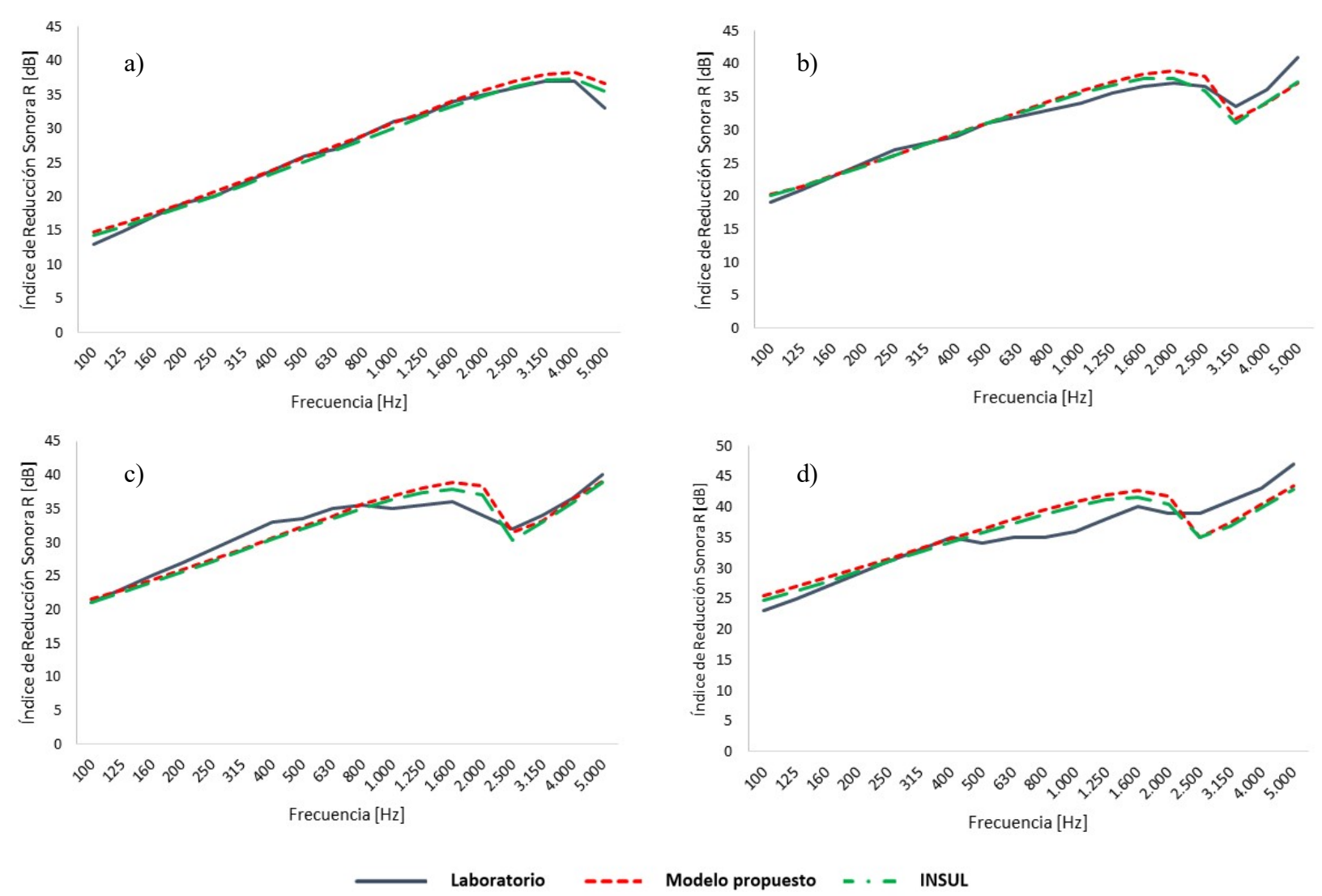

Fig. 2. Comparativa de los índices de reducción sonora entre la medición de laboratorio, la predicción del modelo propuesto y el programa INSUL para paneles multicapa de placas de yeso compuestos por: a) $6,4+6,4 \mathrm{~mm}$ b) $12,7+12,7 \mathrm{~mm}$, c) $12,7+15,9 \mathrm{~mm}$ y d) $15,9+12,7+15,9 \mathrm{~mm}$.

\section{ANÁlisis DE LOS RESUltados}

La metodología empleada para comparar los resultados entre ambos modelos consiste en calcular las diferencias absolutas de los índices $R$ por bandas de tercio de octava entre 100 y $5000 \mathrm{~Hz}$, y los parámetros globales $R w, R w+C$ y $R w+C t r$ obtenidos según la norma ISO 717-1. En la primera parte de la comparativa los materiales se dividen en dos grupos: materiales livianos (vidrio, placa de yeso, madera HDF) y un material pesado (hormigón). Se realiza esta división debido a la ubicación de la frecuencia crítica. Para materiales livianos de poco espesor $f_{c}$ se encuentra por encima de $2000 \mathrm{~Hz}$ aproximadamente, mientras que para materiales pesados $f_{c}$ suele ubicarse por debajo de los 200 $\mathrm{Hz}$.

En las Figs. 3 y 4 se muestran los promedios de desviaciones del índice $R$ en bandas de tercio de octava y de los parámetros globales para ambos grupos de materiales.

En la Fig. 5 se muestran los promedios de desviaciones del índice $R$ en bandas de tercio de octava y de los parámetros globales para los cuatro paneles multicapa de placas de yeso.

\section{DISCUSIÓN}

De la comparativa del promedio de desviaciones absolutas en los indicadores globales $R w(C, C t r)$, los resultados para el caso de los distintos materiales livianos muestran un desvío promedio por debajo de $1 \mathrm{~dB}$.
En el caso del promedio de desviaciones del índice de reducción sonora $R$ para cada banda de tercio de octava entre 100 y $5000 \mathrm{~Hz}$, la diferencia es de 0,5 a 2,5 dB según la frecuencia. El modelo propuesto presenta una mejor aproximación que el programa INSUL para predecir el índice de reducción sonora de materiales livianos de poco espesor.

Los resultados para el caso del hormigón como material pesado muestran un desvío promedio de 0,5 a 1,5 dB en los indicadores globales y para el promedio de diferencias de $R$ en bandas de tercios de octava la diferencia es de 0,5 a 4,5 dB según la frecuencia. En este caso el modelo presenta una mejor aproximación para los indicadores globales según se observa en la Fig. 4b.

En el caso de los paneles multicapa a base de placas de yeso, los resultados muestran que el modelo obtiene una buena aproximación para identificar la frecuencia crítica equivalente del sistema y para predecir el índice de reducción sonora en gran parte del espectro por debajo de esta frecuencia.

A medida que se agregan capas se observa un cambio en la pendiente por debajo de $f_{c, e q}$, produciéndose una desviación que puede apreciarse en la Fig. 5a entre 500 y $4000 \mathrm{~Hz}$. En este caso la diferencia es de 0,5 y $3 \mathrm{~dB}$. Comparando el modelo propuesto y el programa INSUL, existe una diferencia entre 1000 y $2500 \mathrm{~Hz}$ que es levemente menor para el programa comercial. Estas también 


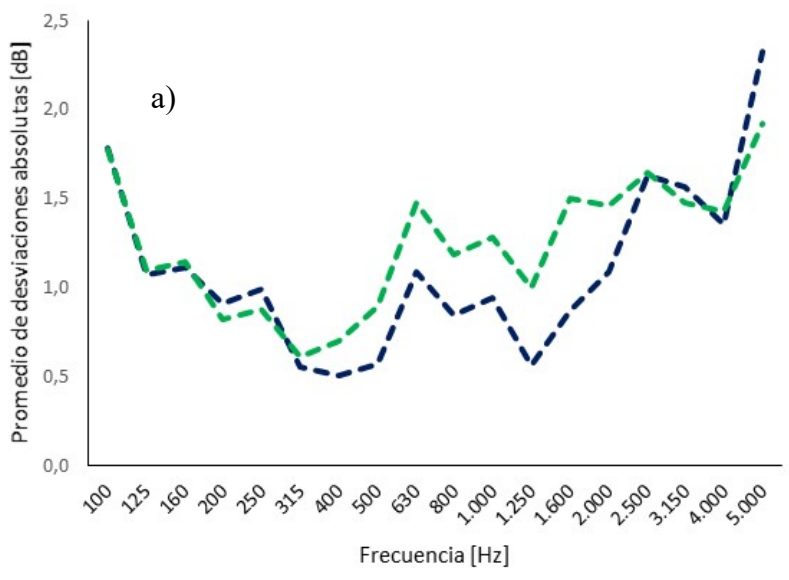

- - Modelo propuesto

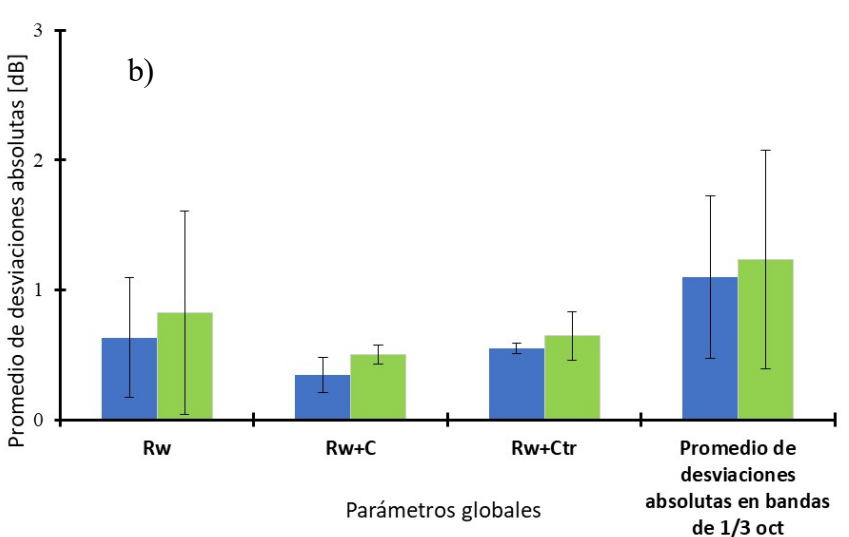

Modelo propuesto

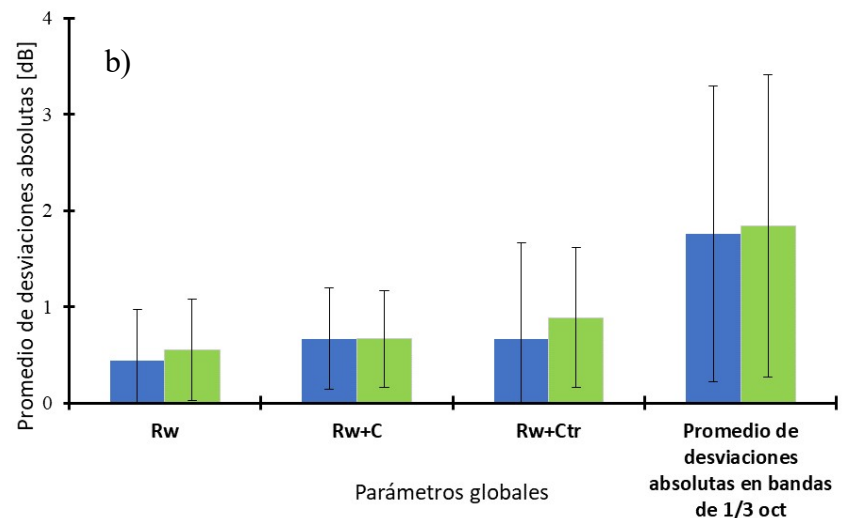

Modelo propuesto

INSUL

Fig. 4. Promedio de desviaciones del modelo propuesto y el programa INSUL para el material pesado: a) por bandas de tercio de octava y b) indicadores globales.

se aprecian en los resultados de los indicadores globales que se muestran en la Fig. 5b. En este caso INSUL tiene mejores resultados respecto al modelo propuesto.

Según Sharp [12], el cambio en la pendiente de $R$ por debajo de $f_{c, e q}$ ocurre cuando la longitud de onda de flexión es comparable a la distancia entre fijaciones, pudiéndose determinar el punto de inflexión a partir de calcular la frecuencia de desacople $f_{D}$, mediante la ecuación:

$$
f_{D}=\frac{2 c_{0}^{2}}{a^{2} f_{c, e q}},
$$

donde $a$ es la distancia en metros entre los montantes de fijación.

En los casos evaluados, $f_{D}$ es igual a $400 \mathrm{~Hz}$ para el panel $6,4+6,4 \mathrm{~mm}$ y $800 \mathrm{~Hz}$ para el panel $12,7+12,7 \mathrm{~mm}$, considerando una separación de 12 pulgadas entre montantes. Para los paneles 12,7+15,9 mm y $15,9+12,7+15,9 \mathrm{~mm}, f_{D}$ es igual a 230 y $240 \mathrm{~Hz}$ respectivamente, siendo la distancia entre montantes de 24 pulgadas.
Si bien sería necesario disponer de un mayor número de ensayos, especialmente para evaluar los casos de paneles multicapa, los resultados obtenidos en esta investigación muestran que el modelo propuesto ofrece una buena precisión en la predicción del índice de reducción sonora $\mathrm{R}$ en todo el rango de frecuencias, tanto para el caso de paneles simples como multicapa.

\section{CONCLUSIONES}

Frente a la ausencia de un modelo de predicción del índice de reducción sonora $\mathrm{R}$ que funcione adecuadamente en todo el rango de frecuencias de interés (de 100 a $5000 \mathrm{~Hz}$ en bandas de tercio de octava), se presenta un modelo basado en la extensión de los modelos de Davy y Sharp para paneles simples a estructuras multicapa, según si se trata de una frecuencia menor o mayor a la frecuencia crítica equivalente. Se adaptan las ecuaciones para el caso de tratarse de paneles formados por capas superpuestas, considerando que se encuentren unidas entre sí mediante fijaciones puntuales. 

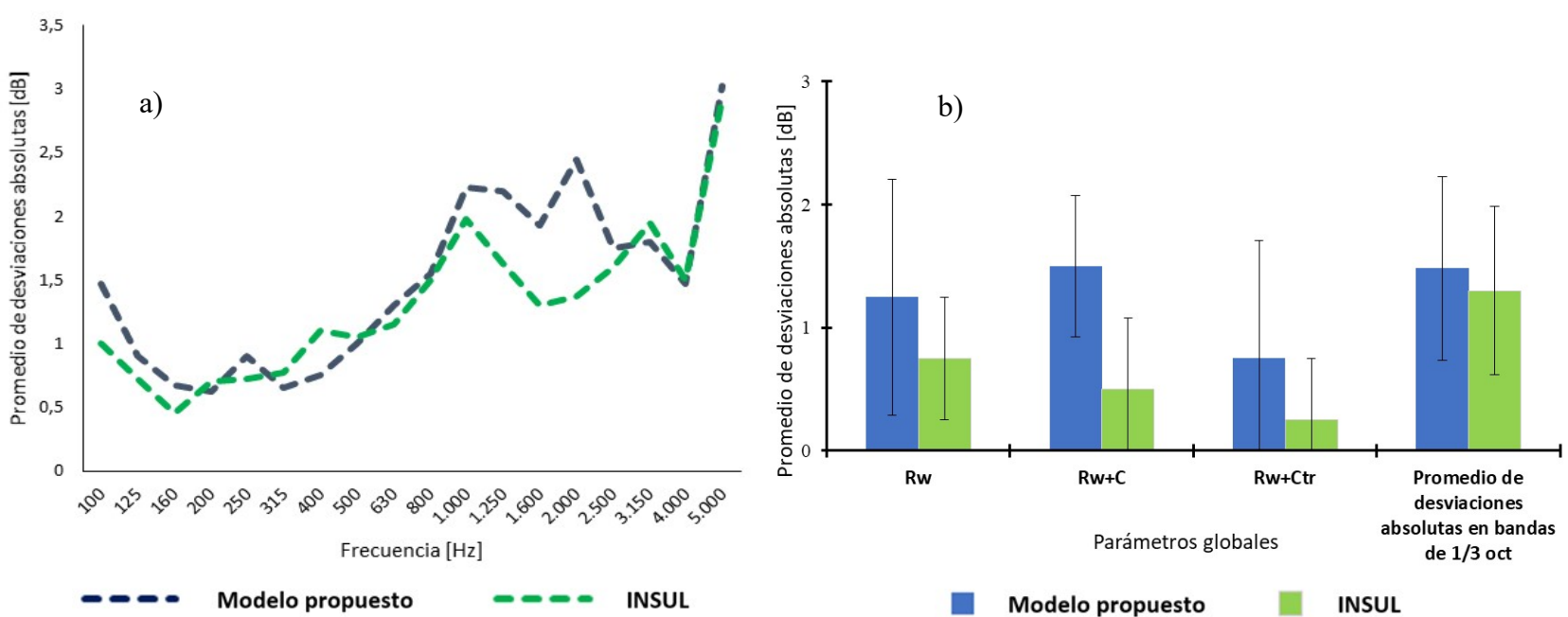

Fig. 5. Promedio de desviaciones absolutas del modelo propuesto y el programa INSUL para el grupo de paneles multicapa: a) por bandas de tercio de octava y b) indicadores globales.

Este modelo se compara respecto a ensayos realizados en laboratorio y respecto a uno de los programas comerciales más conocidos, como es INSUL.

Al no disponer de todos los parámetros físicos de los materiales evaluados, se opta por usar los valores más representativos, sabiendo que se produce un cierto desvío en los resultados obtenidos. También se debe considerar que las propiedades físicas de los materiales usados en la construcción pueden variar de un país a otro, por lo que sería necesario que los fabricantes ofrezcan esta información para mejorar la precisión del modelo.

A partir del análisis de paneles simples homogéneos e isotrópicos y estructuras multicapa compuestas de capas simples unidas entre sí de forma puntual, utilizando el modelo propuesto y el programa comercial INSUL, ambos muestran desviaciones globales pequeñas, del orden de los 2 $\mathrm{dB}$, con respecto a los ensayos disponibles.

\section{AGRADECIMIENTOS}

Esta investigación tiene el apoyo del Programa Científico de la Universidad Nacional Tres de Febrero.

\section{REFERENCIAS}

[1] G. Jäeger, Wiener Akad. Ber. Math-nat. KI 120, Abt. II a, S. 613, 1911.

[2] J.W.S. Rayleigh, The Theory of Sound, vol. 2, page 271, Dover Publications Inc., 1945.

[3] R. Berger, sowie Gesundheits-Ing. 38, 5.49 und 67, T.H. München, 1911.

[4] E. Wintergest, Schalltecknik 4, 5.85, 1931.

[5] V. L. Cremer, "Theorie der schalldämmung dünner Wände beu schrägem einfall," Akustische Zeitschrift VII, Drittes Heft, Siebenter Jahrgang, 1942.

[6] A. London, "Transmission of Reverberant Sound Through Single Walls," Research Paper RP1998, National Bureau of Standards, Volume 42, 1949.

[7] R. Josse y C. Lamure, "Transmissión du son par une paroi simple," Acustica, 14, 266-280, 1964

[8] M. J. Crocker y A. J. Price, "Sound transmission using statistical energy analysis," Journal of Sound and Vibration, 9 (3), 469-486, 1969.

[9] E. C. Sewell, "Transmission of reverberant sound through a singleleaf partition surrounded by an infinite rigid baffle," Journal of Sound and Vibration, 12 (1), 21-32, 1970.
[10] A. Brekke, "Calculation method for the transmission loss of single, double and triple partitions," Applied Acoustics, 14, 225-240, 1981.

[11] B. H. Sharp, "A Study of Techniques to Increase the Sound Insulation of Building Elements," Wyle Laboratories Report, WR 73-5, Wyle Laboratories Research Staff, El Segundo, California. Distribuido como PB222 829, National Technical Information Service, United States Department of Commerce, Springfield, Virginia, 1973.

[12] B. H. Sharp, "Prediction Methods for the Sound Transmission of Building Elements," Noise Control Engineering, 11 (2), 53-63, 1978.

[13] H. Arau, "A new contribution to the study of the soud transmission loss of single panels," Fortschritte der Akustik FASE/DAGA'82, vol. 1, 267-270, 1982.

[14] J.L. Davy, "Predicting the sound insulation of walls," Build. Acoust. $16,1-20,2009$.

[15] J.L. Davy, "Predicting the sound insulation of single leaf walls extension of Cremer's model," Acoustical Society of America, DOI 10.1121/1.3206582, 2009.

[16] B.H.S. Sharp y J.W. Beauchamp, "The transmission loss of multilayer structures," Journal of Sound and Vibration, Volume 9, Issue 3, pp. 383-392, 1969

[17] A. London, "Transmission of Reverberant Sound Through Double Walls," Research Paper RP2058, National Bureau of Standards, Volume 44, 1950

[18] Sitio web de INSUL, Marshall Day Acoustics [On line]. Disponible en: http://www.insul.co.nz

[19] Sitio web de dBKaisla, Ingeniería para el Control del Ruido [On line]. Disponible en: http://dbkaisla.com

[20] C. Hansen, ENC: Software de cálculo de aislamiento acústico Universidad de Adelaide, Australia [On line]. Disponible en: http://www.causalsystems.com.

[21] Sitio web de SoundFlow, Ahnert Feistel Media Group [On line] Disponible en: http://soundflow.afmg.eu.

[22] S. Kurra, "Comparison of the models predicting sound insulation values of multilayered building elements," Applied Acoustics 73, 575-589, 2012.

[23] F. F. Rudder, "Airborne sound transmission loss characteristics of wood-frame construction," Gen. Tech. Rep. FPL-43. Madison, WI: U.S. Department of Agriculture, Forest Service, Forest Products Laboratory, 1985.

[24] R. E. Halliwell, T. R. T. Nightingale, A. C. C. Warnock y Birta J. A. "Gypsum Board Walls: Transmission Loss Data, Internal Report IRC-IR-761," Institute for Research in Construction, National Research Council of Canada, Ottawa, 1988.

[25] J. Solé, Aislamiento Acústico_Índice R, URSA Insulation [On line]. Disponible en: https://www.ursa.es/es-es/descargas/programas-decalculo/Paginas/calculo-aislamiento-acustico.aspx

[26] Sitio web de Viracon [On line]. Disponible en: https://www.viracon.com/acoustic 
Revista elektron, Vol. 3, No. 1, pp. 16-23 (2019)

[27] ISO 717-1: “Acústica. Evaluación del aislamiento acústico en edificios y de los elementos de construcción. Parte 1: Aislamiento a ruido aéreo," 1997.

[28] ISO 140-3: "Acústica. Medición del aislamiento acústico en los edificios y de los elementos de construcción. Parte 3: Medición en laboratorio del aislamiento acústico al ruido aéreo de los elementos de construcción," 1995.

[29] J. Mansilla, F. Masson, I. Calderón de Palma, L. Pepino y Bender L., "Sound insulation of homogeneous single panels: comparison between real construction materials and several prediction models," 24th International Congress on Sound and Vibration ICSV24, Londres, 2017.

[30] J. L. Davy, "The radiation efficiency of finite size flat panels," Acoustics 2004, Transportation Noise and Vibration-The New Millennium, Proceedings of the Annual Conference of the Australian Acoustical Society, Gold Coast, Australia, 3-5 November, edited by M. J. Mee, R. J. Hooker, and I. D. M. Hillock, Australian Acoustical Society, Castlemaine, Victoria, Australia, pp. $555-560,2014$

[31] ISO 12354-1: “Acústica de la edificación. Estimación de las características acústicas de sus elementos. Parte 1: Aislamiento acústico del ruido aéreo entre recintos,” 2000. 